\title{
Le Rapport Bouchard : 5 ans après. Un impact certain, inégal... et encore fragile
}

The Rapport Bouchard Five Years Later: A Sure, Yet Uneven and Fragile Impact

\section{El informe Bouchard: 5 años después. Un impacto cierto, desigual... y todavía frágil}

\section{Gilles Bouchard}

Volume 40, numéro 4, octobre-décembre 1994

URI : https://id.erudit.org/iderudit/1033493ar

DOI : https://doi.org/10.7202/1033493ar

Aller au sommaire du numéro

Éditeur(s)

Association pour l'avancement des sciences et des techniques de la documentation (ASTED)

ISSN

0315-2340 (imprimé)

2291-8949 (numérique)

Découvrir la revue

Citer cet article

Bouchard, G. (1994). Le Rapport Bouchard : 5 ans après. Un impact certain, inégal... et encore fragile. Documentation et bibliothèques, 40(4), 205-211. https://doi.org/10.7202/1033493ar
Résumé de l'article

Après avoir précisé dans quelles circonstances il avait accepté de présider le Comité d'étude sur les bibliothèques scolaires, l'auteur analyse et mesure les impacts de cette étude. Tout en évaluant de façon positive les impacts du rapport en 1989, le président du comité affirme que les observateurs concernés reconnaissent la fragilité d'une telle évolution.
Tous droits réservés (c) Association pour l'avancement des sciences et des techniques de la documentation (ASTED), 1994
Ce document est protégé par la loi sur le droit d'auteur. L'utilisation des services d'Érudit (y compris la reproduction) est assujettie à sa politique d'utilisation que vous pouvez consulter en ligne. 


\title{
Le Rapport Bouchard: 5 ans après Un impact certain, inégal... et encore fragile
}

\author{
Gilles Bouchard* \\ Directeur des Services éducatifs \\ Commission scolaire Taillon
}

Après avoir précisé dans quelles circonstances il avait accepté de présider le Comité d'étude sur les bibliothèques scolaires, l'auteur analyse et mesure les impacts de cette étude. Tout en évaluant de façon positive les impacts du rapport en 1989, le président du comité affirme que les observateurs concernés reconnaissent la fragilité d'une telle évolution.

\section{The Rapport Bouchard Five Years Later : A Sure, Yet Uneven and Fragile Impact}

After describing the circumstances that led him to accept the chairmanship of the Comité d'étude sur les bibliothèques scolaires, the author analyses and measures the impact of the report. While the results of the 1989 report are generally positive, he believes that the educational leaders recognize that progress is fragile.

\author{
El informe Bouchard : 5 años después. Un impacto cierto, \\ desigual... y todavía frágil
}

Después de precisar en que circunstancias había aceptado presidir el comité de estudio sobre las bibliotecas escolares, el autor analiza y evalúa los impactos de este estudio. Aunque evalúa de manera positiva los impactos del informe de 1989, el presidente de este informe afirma que los observadores implicados reconocen la fragilidad de tal evolución.

\begin{abstract}
J'étais sceptique mais aussi stimulé. Sceptique, parce que malgré le nombre important de dossiers, de mémoires, de rapports et de monographies venant de tous les milieux depuis 1975 , le développement continue d'être en sérieux danger. Stimulé, parce que plus que jamais j'ai constaté une volonté d'agir à tous les paliers de la hiérarchie scolaire ${ }^{1}$.
\end{abstract}

C'est par ces mots qu'en octobre 1988 j'exprimais mes sentiments devant le mandat que me confiait le ministre de l'Éducation. Valait-il vraiment la peine de consacrer près de 10 mois de carrière professionnelle à un dossier qui avait jusque là suscité peu d'enthousiasme chez les gestionnaires ou éducateurs? Certes, la bibliothèque dans le réseau scolaire québécois faisait partie des meubles. Mais elle avait plus souvent qu'autrement l'apparence d'un vieux meuble oubliè.

Aujourd'hui, cinq ans plus tard, les membres du Comité d'étude reconnaissent combien ils ont apprécié travailler à la réalisation de ce mandat.
Soutenus par la Direction générale des ressources didactiques, encouragés par le Bureau des sous-ministres, supervisés par un ministre de l'Éducation qui avait maintes fois affiché ses couleurs au regard des ressources pédagogiques de la bibliothèque, les membres du comité ont toujours senti, durant leur mandat, que le dossier recevrait une écoute attentive auprès de toutes les instances.

\section{En fut-il vraiment ainsi?}

Mon point de vue sur les impacts du rapport est peut-être influencé par la place privilégiée que j'ai occupée au sein du comité. Mais, j'ai pu constater, notamment au cours des trois années qui ont suivi le dépôt, que le rapport avait suscité l'intérêt des milieux de l'éducation, non seulement au Québec, mais aussi à l'extérieur. Pendant cette période, il y a eu des rencontres avec la presse francophone et anglophone du Canada et une excellente couverture de presse écrite et parlée ainsi que des revues professionnelles. Je fus conférencier invité à des colloques, cours universitaires, panels et entrevues. Par- tout, on disait apprécier la justesse de l'information, la pertinence et le réalisme des recommandations. J'avais donc nettement l'impression que le rapport servirait de coup de pouce à tous les milieux désirant redresser leur situation relative au développement de la bibliothèque scolaire. Mon évaluation pouvant ne pas être objective, j'ai réuni dernièrement les membres du Comité d'étude. Afin de confirmer ou d'infirmer nos points de vue, il fut décidé de questionner cent trente-cinq directions des Services éducatifs pour évaluer, dans leur commission scolaire respective, les impacts du rapport. Quatre-ving-treize de ces dernières ont accepté de répondre aux quatorze questions et ont rédigé 432 commentaires relatifs à

* L'auteur fut le président du Comité d'étude en 1988 1989.

1. Québec, Comité d'étude sur les bibliothèques scolaires, Les bibliothèques scolaires québécoises: plus que jamais...rapport, Québec, Ministère de l'éducation, Direction générale de l'évaluation et des ressources didactiques, 1989, p. ix. 


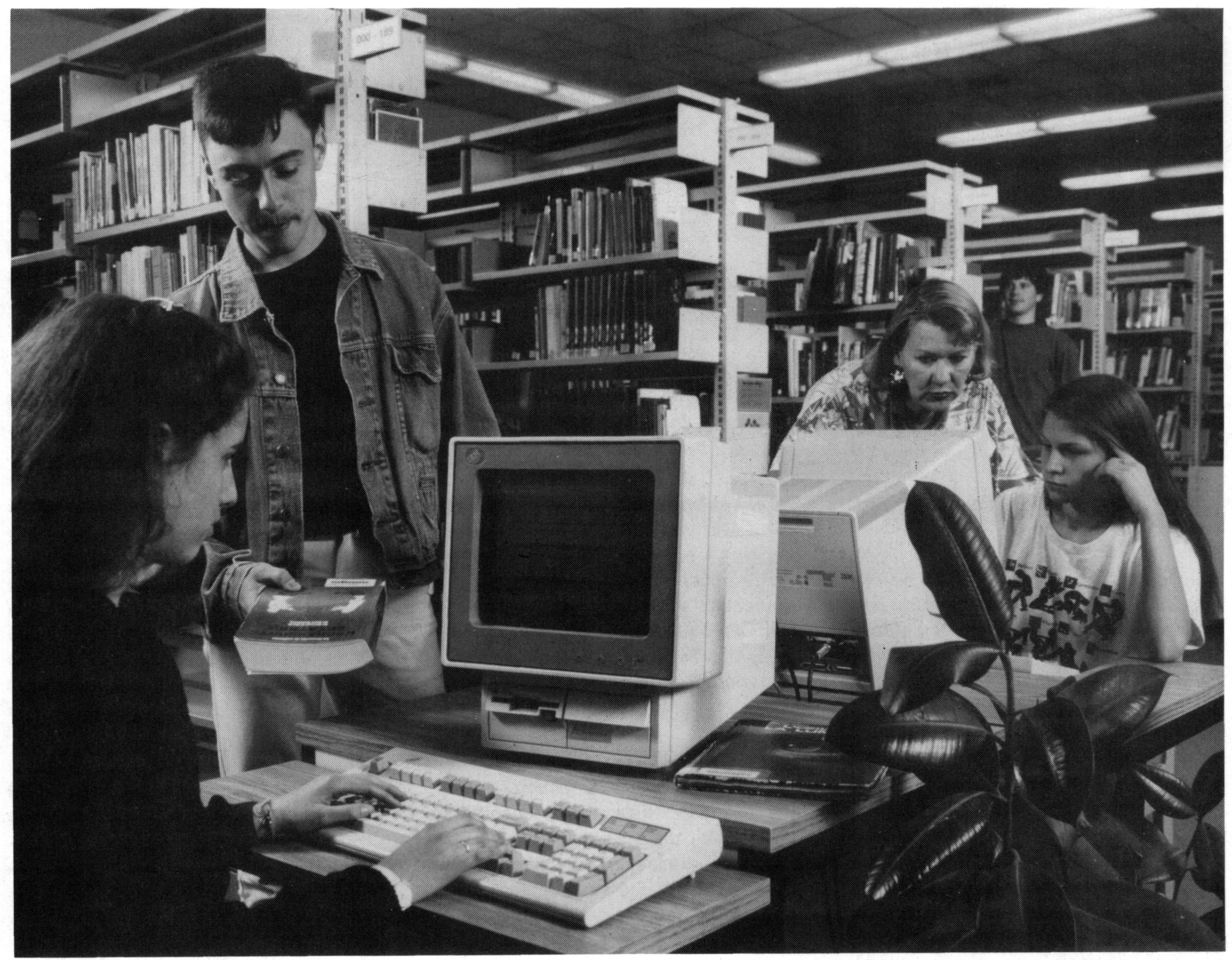

l'évolution de leur réseau de bibliothèques depuis les cinq dernières années. Le présent bilan s'appuie donc sur les données recueillies lors de cette évaluation sommaire (voir l'annexe). Certaines données des banques PERCOS ${ }^{2}$ et PEC ${ }^{3}$ confirment à l'occasion l'évaluation des directions des Services éducatifs.

\section{Un impact certain}

On peut reconnaîtreaujourd'hui, sans aucune hésitation, de nombreuses conséquences heureuses au rapport de 1989. Dès son dépôt, le dossier est devenu une préoccupation ministérielle. Le ministre de l'Éducation de l'époque, Claude Ryan, en a fait un des objets importants de son "discours de la rentrée» à l'automne 1989. La bibliothèque scolaire, faut-il le rappeler, était un des "faits saillants» du discours du Trône du premier ministre à l'ouverture de la session parlementaire d'octobre de la même année. Du côté des commissions scolaires le rapport a aussi provoqué quelques réflexions. L'Association des directeurs généraux du Québec mandatait son comité pédagogique pour analyser les recommandations du rapport. De son côté, le président du Comité d'étude rencontrait dans différentes régions administratives, quelque 300 directions d'école réunies en colloque dont la thématique était la bibliothèque et les ressources documentaires. La presse écrite, notamment L'Actualité, La Presse, The Gazette, Le Devoir en ont fait des dossiers ou éditoriaux percutants quant à l'urgence du «coup de barre» nécessaire.

Cette préoccupation et cette volonté ont engendré des actions concrètes. Près de $70 \%$ des répondants ont confirmé que leur commission scolaire a pris connaissance du rapport et a utilisé la typologie des ressources et des services de la bibliothèque présentée au premier chapitre afin d'analyser l'état de leurs bibliothèques. De ces analyses ont découlé des plans d'action dans près de $57 \%$ des cas selon les réponses obtenues des directions des Services éducatifs. Parfois modestes, parfois spectaculaires, les mesures proposées ont suivi le modèle inspiré par le rapport. Élagage, analyse des besoins, acquisition d'une documentation actualisée, organisation matérielle, animation pédagogique se retrouvent en titre aux principaux chapitres de ces plans d'action.

2. PERCOS : Personnel des commissions scolaires

3. PEC : Plan d'enregistrement comptable 
Évidemment, l'allocation supplémentaire relative à la bibliothèque scolaire, soit $10200000 \$$ en trois ans, a grandement favorisé dans plusieurs milieux l'augmentation du budget consacré à la bibliothèque. Certes on aurait espéré plus. Mais soulignons que l'allocation des 3,20 \$/élève utilisée dans certains cas sous forme de "ticket incitateur» aura permis, selon les données recueillies, d'augmenter de $30 \%$ le budget moyen consacré à l'acquisition de documents. De 5,27 \$ qu'elle était en 1989, la moyenne serait passée à environ 7,50 en 1994. Plusieurs reconnaissent avec raison que cette augmentation suffit à peine à compenser l'augmentation du prix du livre. Toutefois, dans plusieurs commissions scolaires (soit près de $30 \%$ ) il faut ajouter au budget annuel, un budget souvent substantiel qui a été accordé à la bibliothèque dans le cadre d'un plan de relance. Ces budgets peuvent varier de $10000 \$$ à $350000 \$$ selon les milieux et les renseignements reçus. De plus, la consultation nous apprend que des budgets additionnels ont été consacrés à l'aménagement, à l'organisation et surtout, à l'informatisation des bibliothèques. Ainsi, de façon générale, on peut confirmer que le budget réservé à la bibliothèque scolaire a augmenté de façon significative dans plus de la moitié des commissions scolaires au cours des cinq dernières années.

Mais, c'est au regard de la classification et notamment de l'informatisation que le récent sondage révèle le redressement le plus significatif. Si en 1989, 39\% des écoles primaires utilisaient le système de classification Dewey, ce sont $63 \%$ des écoles en 1994 qui profitent d'une bibliothèque bien organisée. Au secondaire, la statistique est passée de $88 \%$ à $93 \%$. Ce bond extrêmement important, pour assurer une meilleure utilisation des ressources documentaires, est particulièrement la résultante de l'informatisation, une opération autrefois lourde et coûteuse devenue aujourd'hui simple et accessible. La technologie du disque optique compact (CD-ROM) associée aux logiciels de gestion des bibliothèques a été apprivoisée par les gestionnaires et est vite devenue l'instrument privilégié des responsables des centres de documentation pour des fins de gestion et de référence. On apprend des commissions scolaires répondantes que $37 \%$ des écoles primaires et $65 \%$ des écoles secondaires ont réalisé ou sont en voie de compléter l'informatisation de leurs bibliothèques. Cet instrument nouveau permet déjà d'apprécier une meilleure utilisation de la bibliothèque. À la question: «Quels sont les principaux facteurs qui ont favorisé le développement des bibliothèques dans votre commission scolaire?" $90 \%$ des directions des Services éducatifs, dont les écoles ont réalisé l'informatisation, reconnaissent les conséquences positives d'une telle opération. $\mathrm{Si}$ cet outil permet aux administrateurs une gestion plus simple et combien plus efficace, il aura surtout permis aux pédagogues d'apprécier tout le potentiel des ressources documentaires dans le processus d'apprentissage.

Et pourtant, à cetégard, le rapport de 1989 avec les quelques lignes réservées à l'informatisation était plutôt timide. Dans le contexte d'alors, on croyait l'opération quasi impossible. Équipement lourd et personnel spécialisé, ont fait place à une simplification telle que le néophyte, souvent bénévole au primaire, assisté d'une aide technique, a permis la réalisation d'une telle entreprise. Cette implantation rapide de cette nouvelle technologie est confirmée par les Services Documentaires Multimedia (SDM) qui dénombrent maintenant quatre-vingt-dix-huit commissions scolaires clientes comparativement à quarante-six en 1990 .

Un autre changement perceptible des dernières années est la nature des écrits ou des discours relatifs au dossier des bibliothèques. Autrefois axés sur l'état déplorable des inventaires, l'utilisation gênante des lieux, les statistiques plus décevantes les unes que les autres, les récents écrits et les récentes interventions décrivent davantage les réalisations intéressantes, les expériences prometteuses, les nouveaux services ou les orientations d'un prochain plan d'action. Les sempiternelles lamentations souvent justifiées de la part du personnel des bibliothèques ont fait place à un marketing orienté vers l'animation et la profitable utilisation des ressources documentaires. À cet égard, les productions du milieu sont nombreuses: guides d'animation, guides d'utilisation, recueils de nouveautés, banques d'activités ont été conçus et produits par le personnel professionnel des commissions scolaires. De plus, des regroupements d'énergie, de ressources humaines, de ressources matérielles et financières sont davantage possibles depuis qu'on a compris que le développement ne vient plus d'en haut. Les nombreuses requêtes de décentralisation des décisions vers les commissions scolaires incitent ces dernières à la concertation. Le Centre régional de ressources documentaires (CERRDOC) de la région Mauricie-BoisFrancs est un heureux exemple de prise en charge de développement par le milieu. Les nombreuses réalisations du Groupe de travail en moyens d'enseignement en Montérégie et dans les régions de LavalLaurentides-Lanaudière, dont les colloques thématiques (sciences de la nature, sciences humaines,...) qui réunissent annuellement plus de 300 enseignants participants, suscitent un intérêt nouveau envers l'utilisation des ressources documentaires. Mais l'exemple de regroupement le plus révélateur est sans contredit celui de l'Association du personnel des services documentaires scolaires (APSDS) qui depuis 1989 recrute annuellement près de 300 membres. Ses trois congrès et son bulletin L'Index ont inspiré de nombreux pédagogues et motivé le personnel professionnel et technique de la bibliothèque.

Le Ministère, de son côté, a aussi fait certains de ses devoirs. En plus d'une allocation supplémentaire incitative durement acquise en cette période de compressions budgétaires, la Direction générale des ressources didactiques aura permis la réalisation d'instruments d'animation. Elle a aussi organisé 26 journées de perfectionnement dans les différentes régions administratives. Cette mise à jour du personnel des commissions scolaires, une première au Québec, a été grandement appréciée par près de 1000 participants. La Direction générale des ressources didactiques a aussi contribué au financement de la recension critique en littérature québécoise pour la jeunesse. Cette analyse de notre littérature jeunesse, en plus d'éclairer les responsables des achats, offre à nos auteurs une vitrine importante pour la diffusion de leurs oeuvres dans nos écoles.

Mais pour les membres du comité, ce sont la réponse et les commentaires à la question 13, à savoir: De façon générale, depuis cinq ans, les ressources de la bibliothèque sont-elles davantage exploitées par le personnel enseignant? qui 
représentent la conséquence la plus heureuse au mémoire de 1989 . On y apprend que $69 \%$ des répondants reconnaissent qu'au primaire les ressources documentaires sont maintenant mieux exploitées. Au secondaire, $56 \%$ des réponses confirment une utilisation plus pédagogique de la bibliothèque. Pas étonnant lorsqu'on apprend que $68 \%$ des commissions scolaires ont organisé au primaire une ou des rencontres d'animation relatives à l'exploitation de la bibliothèque. Au secondaire, $51 \%$ des milieux ont aussi vécu de telles rencontres de sensibilisation. Enfin, il me faut souligner que parmi les principaux facteurs qui ont favorisé ce renouveau, en réponse à la question 14, on retrouve audelà de 240 commentaires qui soulignent l'informatisation, l'allocation supplémentaire et... la sensibilisation, conséquence directe du rapport Les bibliothèques scolaires québécoises: plus que jamais de 1989.

\section{Un impact inégal}

Malheureusement, la récente évaluation réalisée par les directions des Services éducatifs nous apprend d'autres réalités moins heureuses. Si le développement des bibliothèques a connu une certaine évolution dans une majorité des commissions scolaires, c'est encore la désolation dans plusieurs milieux. Dans $25 \%$ des questionnaires, on apprend que le budget a diminué depuis cinq ans, dans $28 \%$ des cas le rapport n'est pas connu ou n'a suscité aucune action ou réaction. Dans $33 \%$ des cas au secondaire et $24 \%$ au primaire, on avoue ne pas avoir perçu une meilleure utilisation des ressources documentaires depuis les cinq dernières années. Les commentaires écrits sont encore plus révélateurs que la statistique. Par exemple, dans certains milieux, on dénonce "la bêtise humaine» de décideurs quant à leur attitude envers la bibliothèque. Ailleurs, on constate que les administrateurs ont utilisé l'allocation supplémentaire pour éponger le déficit. On confirme à plusieurs reprises le peu d'intérêt d'une direction d'école ou du personnel enseignant quant au centre de documentation. Les inégalités peuvent se retrouver à l'intérieur d'une même commission scolaire. C'est le fruit d'une décentralisation mal appliquée, dépourvue de vision ou d'orientation de la part des décideurs locaux. Le développement est alors lié à des personnes plutôt qu'à des politiques. Plusieurs croient encore que le «bonheur viendra d'en haut». Pour ces derniers, on attend toujours le Ministère. À cet égard, il est intéressant de percevoir, dans certaines commissions scolaires, que la bibliothèque redevient une préoccupation chaque fois qu'un écrit ou qu' une action provient de l'extérieur, de préférence, bien sûr, du Ministère. En 1989, le rapport a éveillé certaines écoles pendant un certain temps... puis plus rien. Dans ces mêmes milieux, on nous apprend que le récent sondage adressé aux Services éducatifs a suscité de nouveau un questionnement salutaire. Les membres du comité se rappellent qu'en 1989 leur passage dans une région avait provoqué une augmentation substantielle du budget de la bibliothèque dans certaines écoles. Pour ces milieux faudrait-il alors un rapport tous les cinq ans pour assurer un minimum de développement?

Le suivi de certaines autres recommandations du rapport de 1989 a aussi connu des effets inégaux. On se rappelle qu'une section importante était consacrée à la formation du personnel et notamment à la formation des maîtres. Dans la réforme annoncée relative à la formation initiale du personnel enseignant, tous les espoirs étaient permis. Amère déception pour l'auteur de ces lignes qui a assisté au printemps dernier à la présentation des programmes de formation par quatre universités québécoises. Si l'Université de Montréal présente timidement quelques guides sur l'utilisation des médias et des outils d'information, I'UQAM de son côté est complètement muette à cet égard. Pour plusieurs observateurs, cette rencontre nous informait qu'il y avait peu de place laissée aux stratégies d'enseignement, à la méthodologie du travail intellectuel, à la gestion de classe là où la bibliothèque prend toute son importance. II ne faut pas s'étonner quand le Ministère luimême dans sa documentation de réflexion La formation à l'enseignement secondaire général, Orientations et compétences attendues ${ }^{4}$ consacre une seule ligne à la bibliothèque et qui plus est, cette seule ligne est entre parenthèses (cf. page 25). II me faut souligner cependant l'effort de l'École de bibliothéconomie et des sciences de l'information (EBSI) de l'Université de Montréal qui, depuis cinq ans, présente aux futurs professionnels de la documentation, à l'intérieur d'un cours, le processus d'apprentissage véhiculé par les programmes d'études officiels.

Les membres du Comité soulignent aussi de grands vides relatifs à certaines recommandations du rapport. Les relations bibliothèques municipales et scolaires demeurent hésitantes, isolées et servent davantage la cause des compressions budgétaires plutôt qu'une orientation structurée telle que proposée. «La loi du livre ${ }^{* 5}$ continue de maintenir le prix du livre plus élevé pour les bibliothèques québécoises que pour celles hors Québec. Le statut du personnel professionnel demeure cahotique, bien qu'au niveau salarial les bibliothécaires ont enfin rejoint d'autres professionnels pédagogiques des commissions scolaires. Quant au statut d'enseignant bibliothécaire recommandé par le Comité afin d'assurer le lien entre la pédagogie et la documentation, recommandation qui avait soulevé intérêt et passion dans le milieu scolaire, il demeure toujours l'objet de réflexion. Pour ceux qui souhaitent encore que le réseau scolaire québécois, à l'instar de celui de plusieurs pays, s'enrichisse d'un tel statut, ils peuvent toujours se référer à la proposition de la ministre de l'Éducation, Lucienne Robillard, qui recommandait dans sa réforme Faire avancer l'école l'élargissement de la tâche d'enseignant à d'autres fonctions pédagogiques. Enfin, il nous a été difficile d'évaluer les conséquences relatives à l'emploi de personnel spécialisé. L'intégration de commissions scolaires dans certains milieux, la mutation ou la réaffectation de certains professionnels, les changements de statuts, brouillent la statistique. Cependant, à la lecture de la banque PERCOS; on ne perçoit pas de façon générale, de modifications majeures ni à la hausse ni à la baisse quant au nombre de postes professionnels à la

4. Québec, Ministère de l'éducation, Direction générale de la formation et des qualifications, Direction de la formation du personnel scolaire, $\mathrm{La}$ formation à l'enseignement au secondaire général; orientations et compétences attendues, Québec, 1992, 35 p.

5. Loi sur les entreprises québécoises dans le domaine du livre. 
bibliothèque depuis les cinq dernières années. Le nombre de techniciens en documentation aurait quant à lui augmenté selon les données parues dans PERCOS de 1992

\section{Un impact....encore fragile}

Si dans l'ensemble, on peut évaluer de façon positive les impacts du rapport de 1989 , tous les observateurs concernés reconnaissent la fragilité d'une telle évolution. Dans une majorité de commissions scolaires, les recommandations du rapport ont permis d'éveiller, de sensibiliser, de déclencher, de mobiliser... mais c'est souvent "du bout des doigts» que des gestes concrets ont été réalisés. Là où il y a absence de vision ou d'orientation au regard de la bibliothèque, le développement de cette dernière est directement proportionnel aux croyances ou à l'humeur du cadre d'école ou de service. Et la décision récente d'intégrer dans les bases budgétaires l'allocation complémentaire réservée jusque là à la bibliothèque, en inquiète plusieurs. Pour certains, c'est le retour possible aux années 80 , où budget après budget, on a vu les allocations de la bibliothèque faire figure de peau de chagrin. Les priorités locales sont nombreuses et les compressions budgétaires sont devenues coutumières. Nos meilleurs "évangélistes» sont eux-mêmes inquiets. Seront-ils encore capables, seuls, de convaincre leur milieu de l'importance de la documentation dans le monde de l'information dans lequel nous vivons? Serontils encore capables, seuls, de convaincre leur milieu de l'importance de la lecture dans la réussite éducative et dans l'évolution culturelle d'un peuple? Parce qu'en effet, nos meilleurs "vendeurs» se sentent isolés. À la lecture des réponses au questionnaire, on ressent une grande solitude, voiremême un appel au secours. Et on s'explique mal le silence des hautes instances du Ministère à l'égard de ce dossier. On souligne de façon décevante que ces dernières ne se sont jamais prononcées quant aux recommandations du rapport. Dans les années passées et encore aujourd'hui, le Ministère a déjà fait connaître ses couleurs et il continue de le

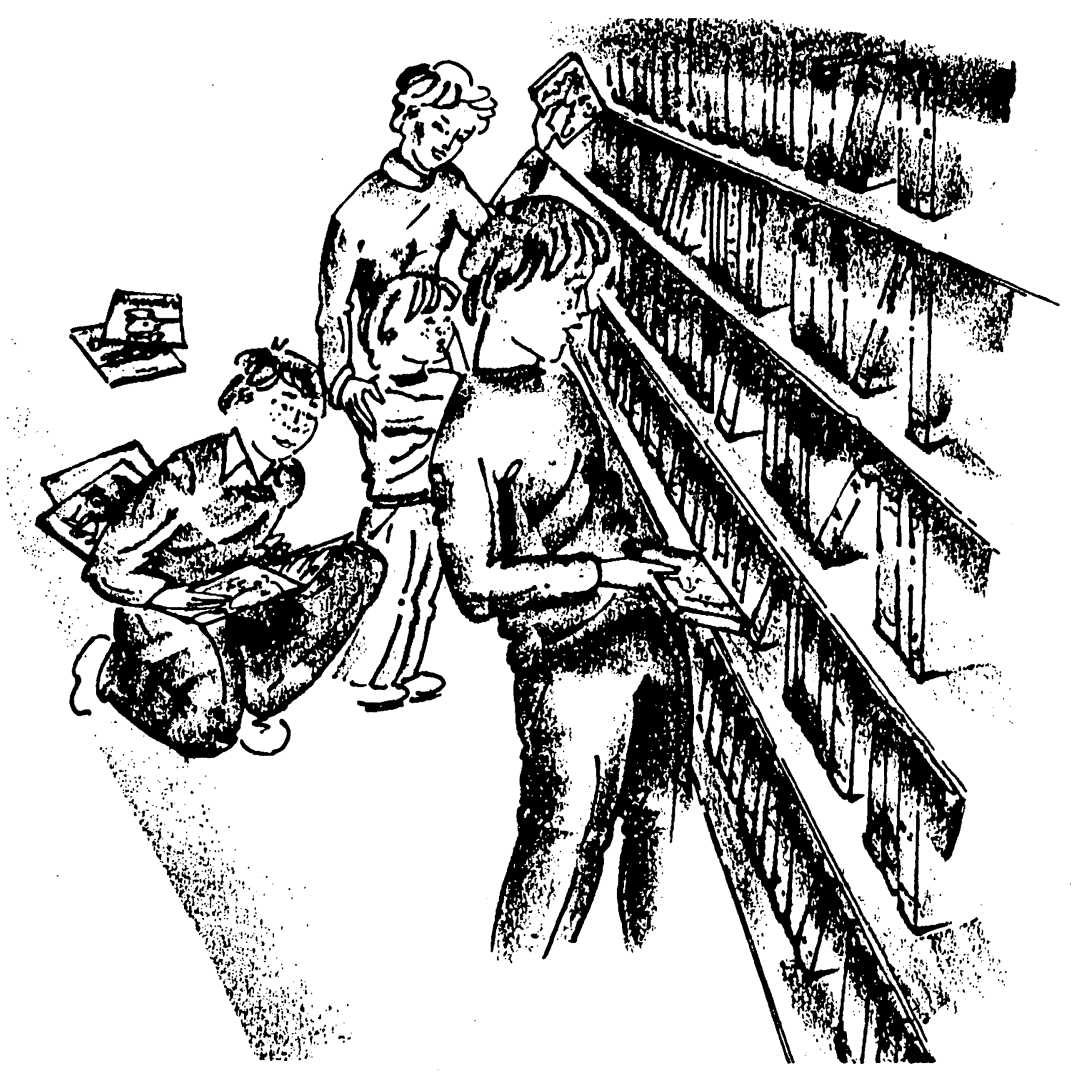

() Ministère de l'éducation du Québec

faire dans l'ensemble des dossiers éducatifs. C'est d'ailleurs le rôle qu'il se reconnaît: «L'État doit mettre l'accent sur les objectifs à poursuivre et sur l'évaluation des résultats de l'ensemble du système éducatif» ${ }^{6}$. Qu'on se rappelle la politique d'évaluation des apprentissages, la politique d'intégration des élèves en difficulté, le plan de développement de la microinformatique, les grandes orientations en matière de formation des maîtres...etc. Mais ce silence quant au développement des bibliothèques est-il symptomatique? Les membres du Comité d'étude attendent encore une réaction, une seule, relative au rapport de 1989. Bien que des hauts fonctionnaires et le ministre luimême aient chaleureusement félicité les auteurs du rapport dans les bureaux ou corridors du Complexe $G$, jamais, non jamais, le Comité n'a reçu de réponse, d'avis, de commentaires écrits, voire même d'accusé de réception d'un dossier qui avait été pourtant une commande officielle du Ministre lui-même.

Certes, le milieu ne s'attend plus à des normes, ratios, directives venant d'en haut. La décentralisation aura ses effets positifs incontestés dans la prise de décision locale. On l'a vu précédemment, plusieurs commissions scolaires ont déjà pris leurs responsabilités en matière de bibliothèque. Mais dans cette période transitoire, où les responsabilités sont déléguées une à une aux gestionnaires locaux, il serait non seulement opportun mais aussi essentiel que des orientations générales servent de cadre de référence aux prochains plans d'action locaux. Sinon, on risque la confusion. Depuis cinq ans, plusieurs pays dont le Japon, la Norvège, la Grande-Bretagne se sont dotés de politique en matière de bibliothèque scolaire et ont permis aux instances locales de se doter de plans d'action locaux cohérents avec le projet éducatif de toute une nation.

Sans cette politique, ou vision commune, le ministre de l'Éducation de l'an 2000 mandatera de nouveau un comité d'étude pour analyser l'état déplorable des bibliothèques des écoles québécoises et continuera sans doute de discourir sur la réussite éducative.

6. Québec, Ministère de l'éducation, Faire avancer l'école; l'enseignement primaire et secondaire québécois: orientations, propositions, questions, Québec, 1993, 39 p. 


\section{ANNEXE}

\section{Synthèse du questionnaire et des réponses obtenues des directions des Services éducatifs des commissions scolaires}

1. De façon générale, dans votre commission scolaire, les directions d'école ont-

Ont répondu oui elles pris connaissance du rapport?

au primaire

au secondaire

$74 \%$

$64 \%$

2. De façon générale, le rapport a-t-il été utilisé pour fin d'analyse de l'état des bibliothèques des écoles de votre commission scolaire?

au primaire

au secondaire

$63 \%$

$57 \%$

3. Le rapport a-t-il permis une sensibilisation du personnel (directions d'école, professionnels, enseignants) sur le rôle de la bibliothèque dans la formation de l'élève?

au primaire

$68 \%$

au secondaire

$56 \%$

4. Sans tenir compte de la subvention du ministère, le budget d'acquisition de documents a-t-il augmenté?

a-t-il diminué?

est-il resté stable?

5. Pour l'année 1993-1994, quel est le budget moyen par élève pour l'acquisition de livres et de périodiques destinés à la bibliothèque?

au primaire

au secondaire

7,00 \$lélève

7,50 \$/élève

6. Depuis 1989 , un plan de développement ou de redressement de la bibliothèque a-t-il été mis en place?

par l'école

par la commission scolaire

Primaire

$26 \%$

$31 \%$

par les deux conjointement

$38 \%$

7. Est-ce que les documents de la collection des bibliothèques sont rangés selon les règles de la classification décimale de Dewey?

au primaire

$63 \%$

au secondaire 
8. Les bibliothèques des écoles sont-elles informatisées?

Ont répondu oui au primaire

$37 \%$

au secondaire

9. Les bibliothèques des écoles sont-elles informatisées

Primaire pour des fins de gestion?

pour des fins de consultation par les élèves?

10. Les écoles ou la commission scolaire ont-elles organisé des rencontres d'animation relatives à l'exploitation pédagogique de la bibliothèque?

au primaire

au secondaire

11. Ya-t-il eu dans votre commission scolaire, une production de matériel d'animation (guides, brochures ou autres)

au primaire

12. Est-ce que le dossier de la «Réussite éducative» a suscité l'émergence de projets relatifs à l'utilisation de la bibliothèque?

au primaire

au secondaire

13. De façon générale, depuis cinq ans, les ressources de la bibliothèque sont-elles davantage exploitées par le personnel enseignant?

au primaire

au secondaire

14. Quels sont, selon vous, les principaux facteurs qui ont favorisé ou défavorisé le développement des bibliothèques dans votre commission scolaire?

De façon générale, les facteurs qui ont favorisé sont:

- l'allocation budgétaire du ministère;

- le Rapport Bouchard;

- l'informatisation;

- le programme de français.

De façon générale, les facteurs qui ont défavorisé sont:

- l'absence de politique;

- les compressions budgéraires;

- le désintéressement des directions d'école et du personnel enseignant. 